\title{
175. Myocardial Action Potentials of Right- and Left-Subepicardial Muscles in the Canine Ventricle and Effects of Manganese Ions
}

\author{
By Akimitsu KamiYama and Yasutake SAEKI \\ Department of Physiology, Yokohama City University School of Medicine, \\ Urafune-cho, Minami-ku, Yokohama, Kanagawa, Japan \\ (Comm. by Yasuji Katsuki, M. J.A., Nov. 12, 1974)
}

The action potentials of ventricular proper muscle in the canine heart were already recorded from the various portions, such as papillary muscles, ${ }^{1)}$ trabecular muscles, ${ }^{2)}$ and muscles of deeper or subepicardial layer. ${ }^{3), 4)}$ Though the electrophysiological properties of the formers have been intensively investigated, informations related to the latter could be rarely found hitherto. The present authors intended first to record the action potentials of subepicardial muscle layer in the right and left ventricles of canine heart and secondly to observe qualitatively the calcium current component in the subepicardial action potential.

Method. Mongrel dogs were anesthetised by the intravenous injection of pentobarbital sodium, $30 \mathrm{mg} / \mathrm{kg}$, and the hearts were immediately removed from the thracic cavities. Muscle strips (about $8 \times 15 \mathrm{~mm}$ ) were excised from epicardial side of free wall in the right and left ventricle, and mounted in a small muscle chamber in which normal Tyrode solution is circulated by a mixed gas of $95 \% \mathrm{O}_{2}$ and $5 \% \mathrm{CO}_{2}$. The half along longitudinal axis of muscle strip was fixed by fine needles on the rubber block, the remainder was kept in hanging in the bathing medium and the free end was connected to mechano-electrical transducer to observe the contractile tension. The action potentials were obtained by a conventional microelectrode technique and displayed simultaneously with tension curve on the cathode ray oscilloscope. The muscle was driven by the electrical pulses at rate of $60 /$ minute and the strength of stimulus was usually about 2 times of threshold. $\mathrm{MnCl}_{2}$ at 0.5 to $3 \mathrm{mM}$ final concentrations were added into the bathing medium to observe the $\mathrm{Ca}^{++}$current component of action potential.

Results. Characteristic action potential was sometimes observed in the right subepicardial muscle, that is action potential showing first and rapid depolarization (phase 0), remarkable phase 1 , and slow and vigorous second depolarization (phase 2). The authors named this potential a spike and dome type action potential 
(Fig. 1, C). The action potentials in the right subepicardial muscle showed more or less remarkable phase 1 and second depolarization, (Fig. 1, A-C), while the action potentials in the left did not show a deep dip between phase 1 and second depolarization, the top of phase 0 transferred immediately to the second depolarization in the most cases (Fig. 1, D-F).

Fig. 2 shows the effects of manganese ions on the subepicardial action potential and on the contractile tension. The action potential
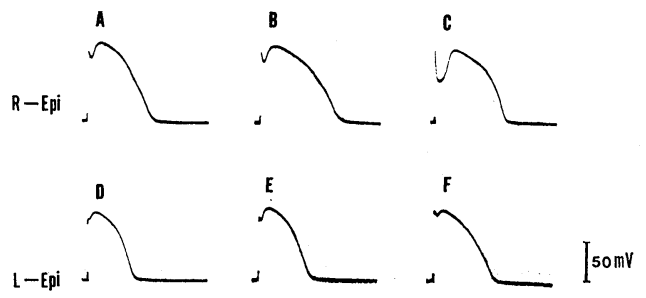

100ms

Fig. 1. Action potentials recorded from right subepicardial muscle (A-C) and from left subepicardial muscle (D-F). R-Epi: right subepicardial muscle. L-Epi: left subepicardial muscle. Spike and dome type action potential in $\mathrm{C}$ was observable as well as $A$ and $B$ in the right subepicardial muscle.

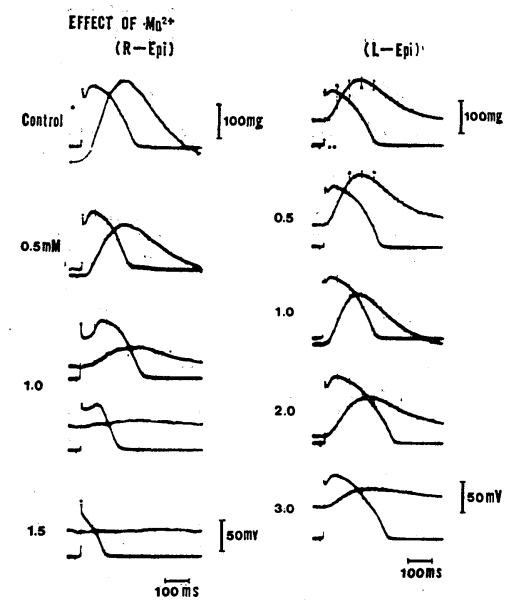

Fig. 2. Effects of manganese ions on the subepicardial muscle. Action potential and mechanical response were simultaneously recorded in each panel. Left column: right subepicardial action potentials and mechanical responses (R-Epi). Right column: left subepicardial action potentials and mechanical responses (L-Epi). Numeral in each panel means millimolar concentration of manganese ions in the bathing medium. 
of right subepicardial muscle was easily eliminated its dome component by the application of manganese ions and contractile tension was simultaneously decreased with the decline of dome component (left column in Fig. 2). On the other hand, the left subepicardial muscle was rather resistive to the effect of manganese ions than the right muscle both on the action potential and on the contractile tension. Similar effect of manganese ions to the left subepicardial muscle was not observable unless the dosage were more than 2 times in the right one (right column in Fig. 2).

The decline of dome component in the spike and dome type action potential was also observed in the right subepicardial muscle preparation immersed into $\mathrm{Ca}^{++}$free Tyrode solution (Fig. 3). In the time course after the replacement from normal Tyrode solution to $\mathrm{Ca}^{++}$ free Tyrode solution, the dome component was gradually eliminated and the decrease of contractile tension run parallel with the decline of dome component.

\section{EFFECT OF - $\mathrm{Ca}^{2+}$ FREE}

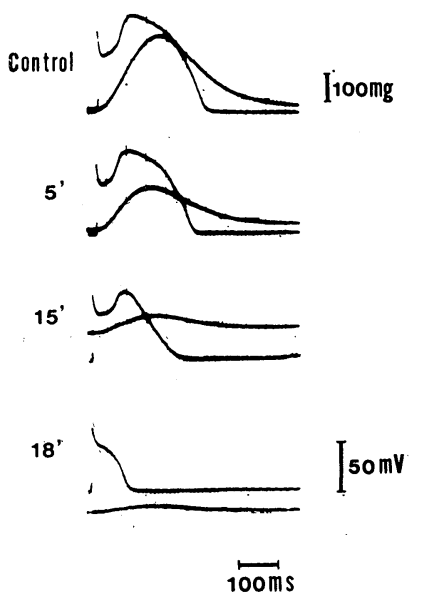

Fig. 3. Effects of $\mathrm{Ca}^{++}$free Tyrode solution on the subepicardial muscle of canine right ventricle. Numerals indicate the time after replacement from normal Tyrode solution to $\mathrm{Ca}^{++}$free Tyrode solution. In each panel, the action potential and the contractile tension curve are simultaneously recorded.

Discussion. Spike and dome type action potential was previously discribed by Matsuda, et al. ${ }^{2}$ It was ascertained that its potential is generated by the special fiber architecture at the Purkinjeventricular fiber junction in the canine ventricle. In the present experiment, subepicardial spike and dome type action potential seems to be generate by the proper nature of muscle cell, because the same 
configuration of action potentials were recorded from every possible insertion on a preparation. Therefore, the authors believe that the hypothesis that the spike and dome type action potential recorded from the Purkinje-ventricular junctional fiber was formed on the basis of special fiber architecture is till alive, and that the results obtained from present experiment can not explain the mechanisms of formation of the "spike and dome" in such junctional fiber.

Recently manganese ion was frequently used as a slow inward current supressor. ${ }^{4)}$ In the present experiment, the second depolarization was abolished by the $\mathrm{Mn}^{++}$and simultaneously contractile tention was also decreased. Moreover, a reduction of extracellular $\mathrm{Ca}^{++}$concentration causes the same change as the application of $\mathrm{Mn}^{++}$to the action potentials. These results may indicate that the dome component, at least the second depolarization, consist mainly of the $\mathrm{Ca}^{++}$ current.

There was a minor difference in the configuration and in the effect of $\mathrm{Mn}^{++}$on the right and on the left subepicardial action potential respectively. The reason of such difference is not clear, but the results suggest that the ionic mechanisms to the action potential formation are slightly different in the right and left subepicardial muscles.

Conclusion. The action potentials of subepicardial muscle layer were recorded from right and left ventricle of the canine heart. In the right, spike and dome type action potential was observed and it was ascertained that its potential was generated by the proper nature of cardiac muscle cell. Moreover, the dome component of action potential was easily eliminated by the $\mathrm{Mn}^{++}$and a contribution of $\mathrm{Ca}^{++}$to the dome component was discussed. A minor differences of the action potential configuration and of the effect of $\mathrm{Mn}^{++}$were recognized between the right subepicardial muscle and the left one, the reason of such differences could not be clear.

\section{References}

1) Hoffman, B. F., and Suckling, E. E.: Amer. J. Physiol., 178, 312 (1953).

2) Matsuda, K., Kamiyama, A., and Hoshi, T.: Electrophysiology and Ultrastructure of the Heart (ed. by T. Sano, V. Mizuhira and K. Matsuda), Grune and Stratton Inc., N. Y. (1967).

3) Matsuda, K., Hoshi, T., and Kameyama, S.: Tohoku J. Exp. Med., 63, 318 (1956).

4) Solberg, L. E., Singer, D. H., Ten Eik, R. E., and Duffin, Jr. E. G.: Circulation Research, 34, 783 (1974).

5) Hagiwara, S., and Nakajima, S.: J. gen. Physiol., 49, 794 (1966). 